International Journal of Pure and Applied Mathematics

Volume 102 No. $4 \quad 2015,593-603$

ISSN: 1311-8080 (printed version); ISSN: 1314-3395 (on-line version)

url: http://www.ijpam.eu

doi: http://dx.doi.org/10.12732/ijpam.v102i4.1

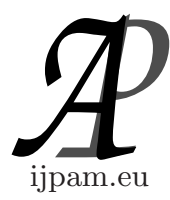

\title{
QUANTUM STOCHASTIC EVOLUTIONS WITH NONLOCAL CONDITION
}

\author{
M.O. Ogundiran \\ Department of Mathematics \\ Obafemi Awolowo University \\ Ile-Ife, NIGERIA
}

\begin{abstract}
This work is concerned with nonlocal problem of Quantum stochastic evolution of Hudson-Parthasarathy quantum stochastic calculus. By employing fixed point theorem we established the existence and uniqueness of mild and strong solutions of the problem.
\end{abstract}

AMS Subject Classification: 34A60, 81S21

Key Words: quantum stochastic processes, fixed point, nonlocal problem

\section{Introduction}

The existence of solution of quantum stochastic differential equations (qsde) of Hudson-Parthasarathy quantum stochastic calculus [9] had been studied by authors in different approach. Amongst these studies is the interesting work [7] in which the problem involving right and left qsde was solved. The mild solution was established for the left qsde while the strong solution was established for the right qsde. But much earlier was the work [6] on the existence of weak solutions of quantum stochastic evolution inclusions arising from a continuous perturbation of quantum stochastic differential inclusions. This work is motivated by the classical form of such evolution equation in which it was observed that problem with nonlocal conditions have much applications than problems with initial conditions. In the classical setting several authors have worked on

Received: October 1, 2014

(C) 2015 Academic Publications, Ltd. url: www.acadpubl.eu 
the nonlocal evolution problem initiated by [4]. Some authors even investigated the nonlocal problems with impulsive effects [1], [2], [3], [5], [11], [8].

Quantum stochastic evolution arising from Hudson-Parthasarathy qsde in this work has an infinitesimal generator $-A$ of a $C_{0}$-semigroup which is a form of the problem considered in [7] and [6]. The present work is a form of generalization of the work [7] with nonlocal conditions and perturbation function. Moreover, by employing a strong locally convex topology in this work, the problem in [6] has been extended to strong case with nonlocal condition. The existence of solutions were established by using a fixed point approach. In Section 2, preliminaries on notations and definitions were stated while main results were established in Section 3.

\section{Preliminaries}

In this subsection we shall introduce the notations and definitions on Quantum stochastic differential equations as applicable in subsequent sections.

\subsection{Notations and Definitions}

To each pair $(D, H)$ consisting of a pre-Hilbert space $D$ and its completion $H$, we associate the set $L_{w}^{+}(D, H)$ of all linear maps $x$ from $D$ into $H$, with the property that the domain of the operator adjoint contains $D$. The members of $L_{w}^{+}(D, H)$ are densely-defined linear operators on $H$ which do not necessarily leave $D$ invariant and $L_{w}^{+}(D, H)$ is a linear space when equipped with the usual notions of addition and scalar multiplication.

Let $\mathbb{D}$ be some pre-Hilbert space whose completion is $\mathcal{R}$ and $\gamma$ is a fixed Hilbert space.

$L_{\gamma}^{2}\left(\mathbb{R}_{+}\right)\left(\right.$resp. $L_{\gamma}^{2}([0, t))$, resp. $\left.L_{\gamma}^{2}([t, \infty)) t \in \mathbb{R}_{+}\right)$is the space of square integrable $\gamma$-valued maps on $\mathbb{R}_{+}$(resp. $[0, t)$, resp. $\left.[t, \infty)\right)$.

The inner product of the Hilbert space $\mathcal{R} \otimes \Gamma\left(L_{\gamma}^{2}\left(\mathbb{R}_{+}\right)\right)$will be denoted by $\langle.,$.$\rangle and \|$.$\| the norm induced by \langle.,$.$\rangle .$

Let $\mathbb{E}, \mathbb{E}_{t}$ and $\mathbb{E}^{t}, t>0$ be linear spaces generated by the exponential vectors in Fock spaces $\Gamma\left(L_{\gamma}^{2}\left(\mathbb{R}_{+}\right)\right), \Gamma\left(L_{\gamma}^{2}([0, t))\right)$ and $\Gamma\left(L_{\gamma}^{2}([t, \infty))\right)$, respectively,

$$
\begin{aligned}
\mathcal{B} & \equiv L_{w}^{+}\left(\mathbb{D} \underline{\otimes} \mathbb{E}, \mathcal{R} \otimes \Gamma\left(L_{\gamma}^{2}\left(\mathbb{R}_{+}\right)\right)\right), \\
\mathcal{B}_{t} & \equiv L_{w}^{+}\left(\mathbb{D} \underline{\otimes} \mathbb{E}_{t}, \mathcal{R} \otimes \Gamma\left(L_{\gamma}^{2}([0, t))\right)\right) \otimes \mathbb{I}^{t} \\
\mathcal{B}^{t} & \equiv \mathbb{I}_{t} \otimes L_{w}^{+}\left(\mathbb{E}^{t}, \Gamma\left(L_{\gamma}^{2}([t, \infty))\right)\right), \quad t>0
\end{aligned}
$$


where $\underline{\otimes}$ denotes algebraic tensor product and $\mathbb{I}_{t}\left(\right.$ resp. $\left.\mathbb{I}^{t}\right)$ denotes the identity map on $\left.\mathcal{R} \otimes \Gamma\left(L_{\gamma}^{2}([0, t))\right)\right)\left(\operatorname{resp} . \Gamma\left(L_{\gamma}^{2}([t, \infty))\right)\right), t>0$.

For every $\xi \in \mathbb{D} \underline{\otimes} \mathbb{E}$ define

$$
\|x\|_{\xi}=\|x \xi\|, \quad x \in \mathcal{B}
$$

then the family of seminorms

$$
\left\{\|\cdot\|_{\xi}: \xi \in \mathbb{D} \underline{\otimes} \mathbb{E}\right\}
$$

generates a strong topology $\tau_{s}$ on $\mathcal{B}$.

The completion of the locally convex spaces $\left(\mathcal{B}, \tau_{s}\right),\left(\mathcal{B}_{t}, \tau_{s}\right)$ and $\left(\mathcal{B}^{t}, \tau_{s}\right)$ are respectively denoted by $\widetilde{\mathcal{B}}, \widetilde{\mathcal{B}}_{t}$ and $\widetilde{\mathcal{B}}^{t}$. The underlying elements of $\widetilde{\mathcal{B}}$ consist of linear maps from $\mathbb{D} \underline{\otimes} \mathbb{E}$ into $\mathcal{R} \otimes \Gamma\left(L_{\gamma}^{2}\left(\mathbb{R}_{+}\right)\right)$having domains of their adjoints containing $\mathbb{D} \underline{\otimes} \mathbb{E}$.

Throughout the paper, for a fixed positive constant $a$, we denote $I$ by $I:=\left[t_{0}, t_{0}+a\right] \subseteq \mathbb{R}_{+}$.

By a stochastic process indexed by $I$, we mean a map $\Phi: I \rightarrow \widetilde{\mathcal{B}}$.

A stochastic process $\Phi$ will be called:

(i) adapted if $\Phi(t) \subseteq \widetilde{\mathcal{B}}_{t}$ for each $t \in I ; \operatorname{Ad}(\widetilde{\mathcal{B}})_{a c}$ denotes the set of adapted absolutely continuous $\widetilde{\mathcal{B}}$-valued stochastic processes.

(ii) locally absolutely $p$-integrable if $t \mapsto\|\Phi(t)\|_{\xi}, t \in I$, lies in $L_{l o c}^{p}(\widetilde{\mathcal{B}})$ for arbitrary $\xi \in \mathbb{D} \underline{\otimes} \mathbb{E}$

The set of all absolutely $p$-integrable stochastic processes will be denoted by $L_{l o c}^{p}(\widetilde{\mathcal{B}})$ and for $p \in(0, \infty), L_{l o c}^{p}(I \times \widetilde{\mathcal{B}})$ is the set of maps $\Phi: I \times \widetilde{\mathcal{B}} \rightarrow \widetilde{\mathcal{B}}$ such that $t \mapsto \Phi(t, x(t)), t \in I$ lies in $L_{\text {loc }}^{p}(\widetilde{\mathcal{B}})$ for every $x \in L_{\text {loc }}^{p}(\widetilde{\mathcal{B}})$.

Consider stochastic processes $E, F, G, H \in L_{l o c}^{2}(I \times \widetilde{\mathcal{B}})$ and $\left(t_{0}, x_{0}\right)$ be a fixed point in $I \times \widetilde{\mathcal{B}}$. Then, a relation of the form

$$
\begin{aligned}
X(t) & =x_{0}+\int_{0}^{t}\left(E(s, X(s)) d \Lambda_{\pi}(s)+F(s, X(s)) d A_{f}(s)\right. \\
& \left.+G(s, X(s)) d A_{g}^{+}(s)+H(s, X(s)) d s \quad t \in[0, T]\right)
\end{aligned}
$$

will be called a stochastic integral equation with coefficients $E, F$, Gand $H$. The stochastic differential equation corresponding to the integral equation above is;

$$
\begin{aligned}
d X(t) & =E(t, X(t)) d \Lambda_{\pi}(t)+F(t, X(t)) d A_{f}(t) \\
& +G(t, X(t)) d A_{g}^{+}(t)+H(t, X(t)) d t \\
X(0) & =x_{0} \quad \text { almost all } t \in[0, T]
\end{aligned}
$$


The notion of solution of (2.1) is defined as follows:

By a solution of $(2.1)$, we mean a stochastic process $\varphi \in \operatorname{Ad}(\widetilde{\mathcal{B}})_{a c} \cap L_{\text {loc }}^{2}(\widetilde{\mathcal{B}})$ such that

$$
\begin{aligned}
d \varphi(t) & =E(t, \varphi(t)) d \Lambda_{\pi}(t)+F(t, \varphi(t)) d A_{f}(t) \\
& +G(t, \varphi(t)) d A_{g}^{+}(t)+H(t, \varphi(t)) d t \text { almost all } t \in I \\
\varphi\left(t_{0}\right) & =\varphi_{0}
\end{aligned}
$$

This work shall be concerned with the existence of mild and strong solutions of nonlocal quantum stochastic evolution problem arising from (2.1), which is of the form

$$
\begin{aligned}
d x(t)+A x(t) d t & =E(t, x(t)) d \Lambda_{\pi}(t)+F(t, x(t)) d A_{f}(t) \\
& +G(t, x(t)) d A_{g}^{+}(t)+H(t, x(t)) d t \quad t \in\left[t_{0}, t_{0}+a\right] \\
x\left(t_{0}\right) & +g\left(t_{1}, \ldots, t_{p}, x(.)\right)=x_{0}
\end{aligned}
$$

where $0 \leq t_{0}<t_{1}<t_{2}<\ldots<t_{p} \leq t_{0}+a(p \in \mathbb{N})$ and $A: D(A) \subset \widetilde{\mathcal{B}} \rightarrow \widetilde{\mathcal{B}}$ is a non-densely defined operator, which is an infinitesimal generator of $C_{0^{-}}$ semigroup $S(t)$, on a locally convex space $\widetilde{\mathcal{B}}$.

\section{Main Results}

A continuous adapted stochastic process $x$ will be said to be a mild solution of problem (2.2) if $x$ is of the integral equation

$$
\begin{aligned}
x(t)= & S\left(t-t_{0}\right) x_{0}+S\left(t-t_{0}\right) h\left(t_{1}, \ldots, t_{p}, x(.)\right) \\
& +\int_{t_{0}}^{t} S(t-s)\left(E(s, x(s)) d \Lambda_{\pi}(s)+F(s, x(s)) d A_{f}(s)\right. \\
& \left.+G(s, x(s)) d A_{g}^{+}(s)+H(s, x(s)) d s\right), \quad t \in I .
\end{aligned}
$$

Theorem 3.1. Assume that:

(i) $0 \leq t_{0}<t_{1}<\ldots<t_{p} \leq t_{0}+a(p \in \mathbb{N})$ and $\Omega=\overline{B(0, r)}=\left\{y:\|y\|_{\xi} \leq\right.$ $r\} \subset \widetilde{\mathcal{B}}$.

(ii) $\Phi \in\{E, F, G, H\} ; \Phi: I \times \widetilde{\mathcal{B}} \rightarrow \widetilde{\mathcal{B}}$ is continuous in $t$ on $I$ and there exist constants $L, L_{\Phi}>0$ with $L=\max \left\{L_{\Phi}\right\}$ such that

$$
\|\Phi(s, y)-\Phi(s, \bar{y})\|_{\xi} \leq L_{\Phi}\|y-\bar{y}\|_{\xi}
$$




$$
\text { for } s \in I ; y, \bar{y} \in \Omega \text {. }
$$

(iii) $h: I^{p} \times \widetilde{\mathcal{B}} \rightarrow \widetilde{\mathcal{B}}$ and there exists a constant $K>0$ such that

$$
\begin{aligned}
\left\|h\left(t_{1}, \ldots, t_{p}, w(.)\right)-h\left(t_{1}, \ldots, t_{p}, \bar{w}(.)\right)\right\|_{\xi} \leq K\|w-\bar{w}\|_{C(I, \Omega)}, & \text { for } w, \bar{w} \in C(I ; \Omega) .
\end{aligned}
$$

(iv) $A$ is the infinitesimal generator of semigroup of contraction $S(t), t \geq 0$, on $\widetilde{\mathcal{B}}$.

$(\mathrm{V})$

$$
\begin{aligned}
M & :=\max _{\tau \in[0, a]}\|U(\tau)\|_{\xi}, \\
N & :=\max \left\{\max _{s \in I}\|E(s, 0)\|_{\xi}, \max _{s \in I}\|F(s, 0)\|_{\xi}, \max _{s \in I}\|G(s, 0)\|_{\xi}, \max _{s \in I}\|H(s, 0)\|_{\xi}\right\}, \\
R & :=\max _{w \in C(I ; \Omega)}\left\|h\left(t_{1}, \ldots, t_{p}, w(.)\right)\right\|_{\xi}
\end{aligned}
$$

(vi) The constants $\left\|x_{0}\right\|_{\xi}, a, r, K, L, M, N$ and $R$ satisfy the following inequalities :

$$
\begin{gathered}
M\left(\left\|x_{0}\right\|_{\xi}+R+r a L+a N\right) \leq r \\
M(K+a L)<1
\end{gathered}
$$

Then problem (2.2) has a unique mild solution $x \in A d_{a c}(I ; \widetilde{\mathcal{B}})$

Proof. Introduce the operator $\Psi$ given by

$$
\begin{aligned}
(\Psi y)(t)= & S\left(t-t_{0}\right) x_{0}-S\left(t-t_{0}\right) h\left(t_{1}, \ldots, t_{p}, y(.)\right) \\
& +\int_{t_{0}}^{t} S(t-s)\left(\left(E(s, y(s)) d \Lambda_{\pi}(s)+F(s, y(s)) d A_{f}(s)\right.\right. \\
& \left.+G(s, y(s)) d A_{g}^{+}(s)+H(s, y(s)) d s\right), \quad t \in I,
\end{aligned}
$$

on the space $\Upsilon:=A d_{a c}(I ; \Omega)$. For an arbitrary $\xi \in \mathbb{D} \underline{\otimes \mathbb{E}}$, we define the norm $\|\cdot\|_{\Upsilon}$ as

$$
\|y\| \Upsilon=\sup \|y(t) \xi\|
$$


We shall show that $\Psi$ maps $\Upsilon$ into $\Upsilon$. We observe that, by (3.5), (3.3), (3.4) and (3.1):

$$
\begin{aligned}
\|(\Psi y)(t)\|_{\xi} & \leq\left\|S\left(t-t_{0}\right) x_{0}\right\|_{\xi}+\left\|S\left(t-t_{0}\right) h\left(t_{1}, \ldots, t_{p}, y(.)\right)\right\|_{\xi} \\
& +\| \int_{t_{0}}^{t} S(t-s)\left(\left(E(s, y(s)) d \Lambda_{\pi}(s)\right.\right. \\
& \left.+F(s, y(s)) d A_{f}(s)+G(s, y(s)) d A_{g}^{+}(s)+H(s, y(s)) d s\right) \|_{\xi} \\
& \leq M\left\|x_{0}\right\|_{\xi}+M R+M \int_{t_{0}}^{t}\left(\|(E(s, y(s))-E(s, 0)) d \Lambda_{\pi}(s)\right. \\
& +(F(s, y(s))-F(s, 0)) d A_{f}(s)+(G(s, y(s))-G(s, 0)) d A_{g}^{+}(s) \\
& +(H(s, y(s))-H(s, 0)) d s) \|_{\xi}+\left(\|E(s, 0)\|_{\xi} d \Lambda_{\pi}(s)\right. \\
& \left.+\|F(s, 0)\|_{\xi} d A_{f}(s)+\|G(s, 0)\|_{\xi} d A_{g}^{+}(s)+\|H(s, 0)\|_{\xi} d s\right) \\
& \leq M\left\|x_{0}\right\|_{\xi}+M R+M \int_{t_{0}}^{t}\left(\left(L_{E}\|y(s)\|_{\xi}+\|E(s, 0)\|_{\xi}\right) d \Lambda_{\pi}(s)\right. \\
& +\left(L_{F}\|y(s)\|_{\xi}+\|F(s, 0)\|_{\xi}\right) d A_{f}(s)+\left(L_{G}\|y(s)\|_{\xi}\right. \\
& \left.+\|G(s, 0)\|_{\xi}\right) d A_{g}^{+}(s) \\
& \left.+\left(L_{H}\|y(s)\|_{\xi}+\|H(s, 0)\|_{\xi}\right) d(s)\right) \\
& \leq M\left(\left\|x_{0}\right\|_{\xi}+R+r a L+a N\right) \leq r \text { for } v \in \Upsilon \text { and } t \in I . \\
&
\end{aligned}
$$

Therefore,

$$
\Psi: \Upsilon \rightarrow \Upsilon
$$

Next we shall show that $\Psi$ is a contraction on $\Upsilon$.

Consider the difference

$$
\begin{aligned}
(\Psi y)(t)-(\Psi \bar{y})(t) & =\int_{t_{0}}^{t} S(t-s)\left[(E(s, y(s))-E(s, \bar{y}(s))) d \Lambda_{\pi}(s)\right. \\
& +(F(s, y(s))-F(s, \bar{y}(s))) d A_{f}(s)+(G(s, y(s)) \\
& -G(s, \bar{y}(s))) d A_{g}^{+}(s) \\
& +(H(s, y(s))-H(s, \bar{y}(s))) d s] \\
& -S\left(t-t_{0}\right)\left[h\left(t_{1}, \ldots, t_{p}, y(.)\right)-h\left(t_{1}, \ldots, t_{p}, \bar{y}(.)\right)\right]
\end{aligned}
$$

for $y, \bar{y} \in \Upsilon$ and $t \in I$ 
Then from (3.7), (3.3), (3.2) and (3.1):

$$
\begin{aligned}
& \|(\Psi y)(t)-(\Psi \bar{y})(t)\|_{\xi} \leq \int_{t_{0}}^{t}\|S(t-s)\|_{\xi}\left[\|(E(s, y(s))-E(s, \bar{y}(s)))\|_{\xi} d \Lambda_{\pi}(s)\right. \\
& +\|(F(s, y(s))-F(s, \bar{y}(s)))\|_{\xi} d A_{f}(s) \\
& +\|(G(s, y(s))-G(s, \bar{y}(s)))\|_{\xi} d A_{g}^{+}(s) \\
& \left.+\|(H(s, y(s))-H(s, \bar{y}(s)))\|_{\xi} d s\right] \\
& +S\left(t-t_{0}\right)\left[h\left(t_{1}, \ldots, t_{p}, y(.)\right)-h\left(t_{1}, \ldots, t_{p}, \bar{y}(.)\right)\right] \\
& \leq M \int_{t_{0}}^{t}\left(L_{E}\left(\|y(s)-\bar{y}(s)\|_{\xi}\right) d \Lambda_{\pi}(s)+L_{F}\left(\|y(s)-\bar{y}(s)\|_{\xi}\right) d A_{f}(s)\right. \\
& \left.+L_{G}\left(\|y(s)-\bar{y}(s)\|_{\xi}\right) d A_{g}^{+}(s)+L_{H}\left(\|y(s)-\bar{y}(s)\|_{\xi}\right) d s\right) \\
& \leq M(K+a L)\|y-\bar{y}\|_{\Upsilon} \text { for } y, \bar{y} \in \Upsilon \text { and } t \in I .
\end{aligned}
$$

If we define $q=M(K+a L)$ then, by (3.8) and (3.4),

$$
\|\Psi y-\Psi \bar{y}\|_{\Upsilon} \leq q\|y-\bar{y}\|_{\Upsilon} \quad \text { for } y, \bar{y} \in \Upsilon
$$

with $0<q<1$. This shows that operator $\Psi$ is a contraction.

Consequently, by (3.6) and (3.9), operator $\Psi$ has a unique fixed point in $\Upsilon$ which is the mild solution of the problem (2.2) $I$.

An adapted stochastic process $x$ is said to be a strong solution of problem (2.2) on $I$, if $x$ is differentiable almost everywhere on $I$ with its derivative $x^{\prime}$ belongs to $L^{1}\left(t_{0}, t_{0}+a ; \widetilde{\mathcal{B}}\right), x\left(t_{0}\right)=x_{0}+h\left(t_{1}, \ldots, t_{p}, x().\right)$ and

$$
\begin{aligned}
d x(t) & =E(t, x(t)) d \Lambda_{\pi}(t)+F(t, x(t)) d A_{f}(t) \\
& +G(t, x(t)) d A_{g}^{+}(t)+H(t, x(t)) d t \text { a.e.on } I
\end{aligned}
$$

Theorem 3.2. Assume that:

(i) $0 \leq t_{0}<t_{1}<\ldots<t_{p} \leq t_{0}+a(p \in \mathbb{N})$ and $\Omega=\overline{B(0, r)}=\left\{y:\|y\|_{\xi} \leq\right.$ $r\} \subset \widetilde{\mathcal{B}}$.

(ii) $\Phi \in\{E, F, G, H\} ; \Phi: I \times \widetilde{\mathcal{B}} \rightarrow \widetilde{\mathcal{B}}$ there exist constants $C, C_{\Phi}>0$ with $C=\max \left\{C_{\Phi}\right\}$ such that

$$
\|\Phi(s, y)-\Phi(s, \bar{y})\|_{\xi} \leq C_{\Phi}\left(|s-\bar{s}|+\|y-\bar{y}\|_{\xi} \quad \text { for } \quad s, \bar{s} \in I ; y, \bar{y} \in \Omega\right.
$$


(iii) $h: I^{p} \times \widetilde{\mathcal{B}} \rightarrow \widetilde{\mathcal{B}}, h\left(t_{1}, \ldots, t_{p},().\right) \in D(A)$ and there exists a constant $K>0$ such that

$$
\begin{array}{r}
\left\|h\left(t_{1}, \ldots, t_{p}, w(.)\right)-h\left(t_{1}, \ldots, t_{p}, \bar{w}(.)\right)\right\|_{\xi} \leq K\|w-\bar{w}\|_{C(I, \Omega)}, \\
\text { for } w, \bar{w} \in C(I ; \Omega) .
\end{array}
$$
on $\widetilde{\mathcal{B}}$

(iv) $A$ is the infinitesimal generator of semigroup of contraction $S(t), t \geq 0$,

$M:=\max _{\tau \in[0, a]}\|S(\tau)\|_{\xi}$,

$N:=\max \left\{\max _{s \in I}\|E(s, 0)\|_{\xi}, \max _{s \in I}\|F(s, 0)\|_{\xi}, \max _{s \in I}\|G(s, 0)\|_{\xi}, \max _{s \in I}\|H(s, 0)\|_{\xi}\right\}$, $R:=\max _{w \in C(I ; \Omega)}\left\|h\left(t_{1}, \ldots, t_{p}, w(.)\right)\right\|_{\xi}$.

(vi) $x_{0} \in D(A)$.

(vii) The constants $\left\|x_{0}\right\|_{\xi}, a, r, K, C, M, N$ and $R$ satisfy the following inequalities :

$$
\begin{gathered}
M\left(\left\|x_{0}\right\|_{\xi}+R+r a C+a N\right) \leq r, \\
M(K+a C)<1 .
\end{gathered}
$$

(viii) $x$ is the mild solution of problem (2.2) on $I$.

Then $x$ is a strong solution of problem (2.2) on $I$.

Proof. Observe that

$$
\begin{aligned}
x(t+h)-x(t) & =\left[S\left(t+h-t_{0}\right) x_{0}-S\left(t-t_{0}\right) x_{0}\right] \\
& -\left[S\left(t+h-t_{0}\right) h\left(t_{1}, \ldots, t_{p}, x(.)\right)-S\left(t-t_{0}\right) h\left(t_{1}, \ldots, t_{p}, x(.)\right)\right] \\
& +\int_{t_{0}}^{t+h} S(t+h-s)\left[E(s, x(s)) d \Lambda_{\pi}(s)+F(s, x(s)) d A_{f}(s)\right. \\
& \left.+G(s, x(s)) d A_{g}^{+}(s)+H(s, x(s)) d s\right] \\
& -\int_{t_{0}}^{t} S(t-s)\left[E(s, x(s)) d \Lambda_{\pi}(s)+F(s, x(s)) d A_{f}(s)\right. \\
& \left.+G(s, x(s)) d A_{g}^{+}(s)+H(s, x(s)) d s\right]
\end{aligned}
$$




$$
\begin{aligned}
& =\left[S\left(t+h-t_{0}\right) x_{0}-S\left(t-t_{0}\right) x_{0}\right] \\
& -\left[S\left(t+h-t_{0}\right) h\left(t_{1}, \ldots, t_{p}, x(.)\right)-S\left(t-t_{0}\right) h\left(t_{1}, \ldots, t_{p}, x(.)\right)\right] \\
& +\int_{t_{0}}^{t+h} S(t+h-s)\left[E(s, x(s)) d \Lambda_{\pi}(s)+F(s, x(s)) d A_{f}(s)\right. \\
& \left.+G(s, x(s)) d A_{g}^{+}(s)+H(s, x(s)) d s\right] \\
& +\int_{t_{0}}^{t} S(t-s)\left[(E(s+h, x(s+h))-E(t, x(s))) d \Lambda_{\pi}(s)\right. \\
& +(F(s+h, x(s+h))-F(s, x(s))) d A_{f}(s) \\
& +(G(s+h, x(s+h))-G(s, x(s))) d A_{g}^{+}(s) \\
& +(H(s+h, x(s+h))-H(s, x(s))) d s], \quad t \in I .
\end{aligned}
$$

Since all the assumptions of Theorem 3.1 are satisfied it is obvious that problem $(2.2)$, possesses a unique mild solution belonging to $C(I ; \widetilde{\mathcal{B}})$ which, according to assumption (ix), is denoted by $x$.

Next, we shall show that this mild solution is a strong solution of problem (2.2) on $I$. Let

$$
\bar{N}:=\max _{s \in I}\|\Phi(s, x(s))\|_{\xi}, \quad \Phi \in\{E, F, G, H\}
$$

Consequently, by (3.13), (3.11), (3.12) and (3.10),

$$
\begin{aligned}
\|x(t+h)-x(t)\|_{\xi} & \leq h M\left\|h x_{0}\right\|_{\xi}+h M\left\|A h\left(t_{1}, \ldots, t_{p}, x(.)\right)\right\|_{\xi}+h M \bar{N} \\
& +M C \int_{t_{0}}^{t}\left(h+\|x(s+h)-x(s)\|_{\xi}\right) d s \\
& \leq h M\left\|A x_{0}\right\|_{\xi}+h M\left\|A h\left(t_{1}, \ldots, t_{p}, x(.)\right)\right\|_{\xi}+h M \bar{N} \\
& +M C a h+M C \int_{t_{0}}^{t}\|x(s+h)-x(s)\|_{\xi} d s \\
& =\bar{C} h+M C \int_{t_{0}}^{t}\|x(s+h)-x(s)\|_{\xi} d s, \quad t \in I,
\end{aligned}
$$

where

$$
\bar{C}:=M\left[\left\|A x_{0}\right\|_{\xi}+\left\|A h\left(t_{1}, \ldots, t_{p}, x(.)\right)\right\|_{\xi}+\bar{N}+a C\right]
$$

From (3.14) and Gronwall's inequality,

$$
\|x(t+h)-x(t)\|_{\xi} \leq \bar{C} e^{a M C} h, \quad t \in I
$$


and therefore $x$ is Lipschitz continuous on $I$.

The Lipschitz continuity of $x$ on $I$ combined with the Lipschitz of $E, F, G, H$ on $I \times \widetilde{\mathcal{B}}$ implies that $t \rightarrow \Phi(t, x(t))$ is Lipschitz continuous on $I$. This property of $\Phi$ together with the assumptions of Theorem 3.1 that the linear Cauchy problem

$$
\begin{aligned}
d y(t) & =A y(t)+E(t, x(t)) d \Lambda_{\pi}(t)+F(t, x(t)) d A_{f}(t) \\
& +G(t, x(t)) d A_{g}^{+}(t)+H(t, x(t)) d t \text { a.e.on } I \\
y\left(t_{0}\right) & =x_{0}-h\left(t_{1}, \ldots, t_{p}, u(.)\right)
\end{aligned}
$$

which can be written in the equivalent form has a unique strong solution $y$ on $I$ satisfying the equation

$$
\begin{aligned}
y(t) & =S\left(t-t_{0}\right) x_{0}-S\left(t-t_{0}\right) h\left(t_{1}, \ldots, t_{p}, x(.)\right) \\
& +\int_{t_{0}}^{t} S(t-s)\left[E(s, x(s)) d \Lambda_{\pi}(s)+F(s, x(s)) d A_{f}(s)\right. \\
& \left.+G(s, x(s)) d A_{g}^{+}(s)+H(s, x(s)) d s\right]=x(t) \quad t \in I .
\end{aligned}
$$

Consequently, $x$ is a strong solution of problem (2.2) on $I$ and therefore the proof of Theorem 3.2 is complete.

\section{References}

[1] A. Anguraj and M. Mallika Arjunan, Existence and uniqueness of mild and classical solutions of impulsive evolution equations, Electronic Journal of Differential Equations, 111 (2005), 1-8.

[2] M. Malika Arjunan, V. Kavitha and S. Selvi, Existence results for impusive differential equations with nonlocal conditions via measures of noncompactness, J. Nonlinear Sci. Appl., 5 (2012), 195-205.

[3] M. Benchohra and S. K. Ntouyas, Existence of mild solutions of semilinear evolution inclusions with nonlocal conditions, Georgian Math. J., 7 (2000), 221-230.

[4] L. Byszewski, Theorems about the existence and uniqueness of solutions of a semilinear evolution nonlocal Cauchy problem, J. Mth. Anal. Appl. 162 (1991) 494-505, doi: 10.1016/0022-247X(91)90164-U. 
[5] L. Byszewski and V. Lakshmikantham, Theorem about the existence and uniqueness of solutions of nonlocal Cauchy problem in a Banach space, Appl. Anal., 40 (1990), 11-19, doi: 10.1080/00036819008839989.

[6] G.O.S. Ekhaguere, Quantum stochastic evolutions, International Journal of Theoretical Physics, 35 (1996) 9, 1909-1946, doi: 10.1007/BF02302422.

[7] F. Fagnola and S.J. Wills, Solving quantum stochastic differential equations with unbounded coefficients, J. Funct. Anal., 198 (2003) 279-310, doi: 10.1016/S0022-1236(02)00089-7.

[8] Z. Fan, Impulsive problems for semilinear differential equations with nonlocal conditions, Nonlinear Anal., 72 (2010), 1104-1109, doi: 10.1016/j.jfa.2009.10.023.

[9] R.L. Hudson and K.R. Parthasarathy, Quantum Ito's formula and stochastic evolutions, Comm. Math. Phys., 93, No. 3 (1984), 301-323, doi: 10.1007/BF01258530.

[10] M. O. Ogundiran and V. F. Payne, On the existence of solutions of impulsive quantum stochastic differential equations, Differential Equations and Control Processes, 2 (2013), 62-73.

[11] X. Xue, Existence of semilinear differential equations with nonlocal initial conditions, Acta Math. Sinica, 23, No. 6 (2007), 983-988, doi: 10.1007/s10114-005-0839-3. 
